\section{OPEN ACCESS}

Edited by: Giovanni Piccoli,

University of Trento, Italy

Reviewed by:

Enrico Domenici,

University of Trento, Italy

Gianluca Serafini,

San Martino Hospital, University of

Genoa, Italy

${ }^{*}$ Correspondence:

Mari-Anne Philips marianne.philips@ut.ee; maphilips@gmail.com

Received: 26 October 2017 Accepted: 08 January 2018

Published: 29 January 2018

Citation:

Karis K, Eskla K-L, Kaare M, Täht K, Tuusov J, Visnapuu T, Innos J, Jayaram $M$, Timmusk $T$,

Weickert CS, Väli M, Vasar E and Philips M-A (2018) Altered Expression Profile of IgLON Family of Neural Cell Adhesion Molecules in the Dorsolateral Prefrontal Cortex of Schizophrenic Patients.

Front. Mol. Neurosci. 11:8 doi: 10.3389/fnmol.2018.00008

\title{
Altered Expression Profile of IgLON Family of Neural Cell Adhesion Molecules in the Dorsolateral Prefrontal Cortex of Schizophrenic Patients
}

\author{
Karina Karis 1,2, Kattri-Liis Eskla ${ }^{1,2}$, Maria Kaare ${ }^{1,2}$, Karin Täht ${ }^{3}$, Jana Tuusov ${ }^{4,5}$, \\ Tanel Visnapuu 1,2, Jürgen Innos ${ }^{1,2}$, Mohan Jayaram ${ }^{1,2}$, Tónis Timmusk ${ }^{6}$, \\ Cynthia S. Weickert 7,8 , Marika Väli ${ }^{4,5}$, Eero Vasar ${ }^{1,2}$ and Mari-Anne Philips ${ }^{1,2 *}$
}

${ }^{1}$ Department of Physiology, Institute of Biomedicine and Translational Medicine, University of Tartu, Tartu, Estonia, ${ }^{2}$ Centre of Excellence for Genomics and Translational Medicine, University of Tartu, Tartu, Estonia, ${ }^{3}$ Institute of Psychology, University of Tartu, Tartu, Estonia, ${ }^{4}$ Department of Pathological Anatomy and Forensic Medicine, University of Tartu, Tartu, Estonia, ${ }^{5}$ Estonian Forensic Science Institute, Tallinn, Estonia, ${ }^{6}$ Department of Chemistry and Biotechnology, Tallinn University of Technology, Tallinn, Estonia, ${ }^{7}$ Faculty of Medicine, School of Psychiatry, University of New South Wales, Sydney, NSW, Australia, ${ }^{8}$ Schizophrenia Research Institute, Neuroscience Research Australia, Randwick, NSW, Australia

Neural adhesion proteins are crucial in the development and maintenance of functional neural connectivity. Growing evidence suggests that the IgLON family of neural adhesion molecules LSAMP, NTM, NEGR1, and OPCML are important candidates in forming the susceptibility to schizophrenia (SCZ). IgLON proteins have been shown to be involved in neurite outgrowth, synaptic plasticity and neuronal connectivity, all of which have been shown to be altered in the brains of patients with the diagnosis of schizophrenia. Here we optimized custom 5'-isoform-specific TaqMan gene-expression analysis for the transcripts of human IgLON genes to study the expression of IgLONs in the dorsolateral prefrontal cortex (DLPFC) of schizophrenic patients $(n=36)$ and control subjects $(n=36)$. Uniform 5 '-region and a single promoter was confirmed for the human NEGR1 gene by in silico analysis. IgLON5, a recently described family member, was also included in the study. We detected significantly elevated levels of the NEGR1 transcript (1.33-fold increase) and the NTM $1 \mathrm{~b}$ isoform transcript (1.47-fold increase) in the DLPFC of schizophrenia patients compared to healthy controls. Consequent protein analysis performed in male subjects confirmed the increase in NEGR1 protein content both in patients with the paranoid subtype and in patients with other subtypes. In-group analysis of patients revealed that lower expression of certain IgLON transcripts, mostly LSAMP $1 \mathrm{a}$ and $1 \mathrm{~b}$, could be related with concurrent depressive endophenotype in schizophrenic patients. Additionally, our study cohort provides further evidence that cannabis use may be a relevant risk factor associated with suicidal behaviors in psychotic patients. In conclusion, we provide clinical evidence of increased expression levels of particular IgLON family members in the DLPFC of schizophrenic patients. We propose that 
alterations in the expression profile of IgLON neural adhesion molecules are associated with brain circuit disorganization in neuropsychiatric disorders, such as schizophrenia. In the light of previously published data, we suggest that increased level of NEGR1 in the frontal cortex may serve as molecular marker for a wider spectrum of psychiatric conditions.

Keywords: schizophrenia, DLPFC, LSAMP, NTM, OPCML, NEGR1, IGLON5, alternative promoters

\section{INTRODUCTION}

Schizophrenia (SCZ) is a severe neuropsychiatric disorder with complex etiology, affecting approximately $1 \%$ of the world's population (van Os and Kapur, 2009; Costain and Bassett, 2012). Schizophrenic patients have a reduced quality of life, significant functional impairment and suicide rates among schizophrenic patients remain alarmingly high (Pompili et al., 2007). High heritability of schizophrenia has been consistently demonstrated by family studies (Mäki et al., 2005) and genome-wide association studies have identified numerous risk chromosome loci candidates for schizophrenia (O'Donovan et al., 2008; Purcell et al., 2009; Stefansson et al., 2009; Ripke et al., 2013, 2014; Yue et al., 2017) indicating many common gene variants providing subtle effects (Giusti-Rodríguez and Sullivan, 2013). Evidence from GWAS and studies revealing differentially expressed proteins in schizophrenia suggests that the majority of the pathophysiological processes underlying schizophrenia are involved in neuronal transmission, synaptic maturation and plasticity, neurite outgrowth and neurogenesis (Hosak et al., 2012; Nascimento and Martins-de-Souza, 2015; Singh et al., 2017).

A growing body of evidence suggests the existence of certain genetically transmitted intermediate phenotypes for psychiatric disorders such as schizophrenia that determine the molecular setup of the neuronal substrate (Gottesman and Gould, 2003). Disrupted functional brain connectivity, reflecting abnormal integrity of neural tracts, has been suggested to be an endophenotype for schizophrenia (Li et al., 2017). Cell membrane adhesion molecules, such as the IgLON proteins, are crucial for the correct assembly of neural circuits. The IgLON superfamily of neural cell adhesion molecules are Gpianchored neural cell surface glycoproteins (Salzer et al., 1996) in the neural cell membrane, including Lsamp (limbic system associated membrane protein), NTM (neurotrimin), OPCML (opioid-binding cell adhesion molecule), Kilon/Negr1 (neuronal growth regulator 1) and IgLON5 (Vanaveski et al., 2017). IgLON proteins form homophilic and heterophilic complexes along the cell surface (Reed et al., 2004) and regulate neurite outgrowth (Akeel et al., 2011), dendritic arborization (Pischedda et al., 2014; Pischedda and Piccoli, 2016) and synapse formation (Hashimoto et al., 2009) both in the developing and adult brain.

Polymorphisms in the human IgLON genes have been linked with a variety of psychiatric conditions, including schizophrenia. Based on Gwas meta-analysis from the Psychiatry Genomics Consortium (Ripke et al., 2014), there is strongest evidence for the association of OPCML and NEGR1 genes with schizophrenia, compared to other IgLONs. The Gwas meta-analysis data from
Ripke et al. (2014) in the genomic regions of IgLONs has been visualized in Supplementary Figures S1-S3. In earlier studies, OPCML gene at 11q25 has been found to be a schizophrenia susceptibility locus in the European (O'Donovan et al., 2008) and Thai populations (Panichareon et al., 2012) and NTM has been significantly associated with schizophrenia in a genome-wide meta-analysis (Wang et al., 2010). Copy number variants in $11 \mathrm{q} 25$ where NTM and OPCML gene lie in close proximity have repeatedly been identified in patients with the diagnosis of schizophrenia (Magri et al., 2010; Ye et al., 2012). The most convincing evidence linking Lsamp with schizophrenia comes from protein studies in the post mortem brains: the level of the LSAMP protein is increased in the postmortem dorsolateral prefrontal cortex (DLPFC) (Behan et al., 2009) and in the synaptosome fraction of the orbitofrontal cortex (Velásquez et al., 2017) in patients with schizophrenia. Furthermore, Lsamp and NEGR1 proteins are significantly upregulated in the post-mortem anterior prefrontal cortex of the patients with schizophrenia compared to healthy controls (Cox et al., 2016). An association between schizophrenia and polymorphisms in the Lsamp gene has been shown in the European (Koido et al., 2014) and Chinese (Chen et al., 2017) subjects.

In our previous study (Vanaveski et al., 2017) we have shown a twin-promoter structure in NTM and OPCML, similar to the genomic organization reported for LSAMP (Pimenta and Levitt, 2004), characterized by two alternative promotors $1 \mathrm{a}$ and $1 \mathrm{~b}$, whereas mouse Negr1 and Iglon5 transcripts have a uniform $5^{\prime}$ region, suggesting a single promoter. As the human NEGR1 gene has been expected to have putative alternative promoters in public databases, Ensembl ${ }^{1}$ and NCBI-aceview ${ }^{2}$, in silico analysis of the genomic structure of human NEGR1 gene was performed before the experimental part of the current study for the optimal design of gene-expression assays.

The main focus of the study was to determine whether patients with schizophrenia express altered mRNA/protein levels of IgLON genes compared to controls in a well characterized cohort (Weickert et al., 2010). We examined the DLPFC (Brodmann area 46) as this region is important in the neuropathology of schizophrenia due to its role in executive cognitive functions (Harrison and Weinberger, 2005; Baumgartner et al., 2011; Catts et al., 2013). Alternative transcripts derived from 1a to $1 \mathrm{~b}$ promoters of LSAMP, NTM, and OPCML were quantified separately by using a custom $5^{\prime}$-isoform-specific TaqMan geneexpression assay. As suicide is a major cause of death among patients with schizophrenia (Pompili et al., 2007), we also studied

\footnotetext{
${ }^{1}$ www.ensembl.org

${ }^{2}$ https://www.ncbi.nlm.nih.gov
} 
gene expression changes and other significant factors, such as substance abuse, in patients who committed suicide attempts or completed suicide compared with patients with no suicidal behaviors.

\section{MATERIALS AND METHODS}

\section{In Silico Analysis of Alternative First Exons of Human NEGR1 Gene}

Bioinformatic analysis of alternative first exons and respective upstream regions ( $5^{\prime} \mathrm{UTR}$ ) of human NEGR1 transcript was performed along with all ESTs present and with the LSAMP gene. All the alignments were performed with Clustal Omega Multiple sequence alignment service ${ }^{3}$. Alternative transcripts were further analyzed by mapping representative EST sequences to the NEGR1 transcript and genome region. High quality ESTs spanning upstream of I or II exon were subsequently aligned to explore potential existence of alternative first exons. The available transcripts query was confirmed by BLAST search in NCBI Refseq and Non-Refseq databases. The putative transcriptional start site was analyzed using DBTSS database ${ }^{4}$. The sequences used in the current study were obtained from respective databases in Ensembl (release 89) or NCBI. The sequence identity numbers were obtained from Ensembl database and accession codes from NCBI RefSeq. The sequences for the multiple sequence alignment were procured from the NCBI site. The coding sequences in the FASTA format were used for the alignment. CLUSTALW multiple sequence alignment program was used for aligning the sequences for OPCML1a (NM_001012393.1), OPCML1b (NM_002545.3) NTM 1a (NM_001048209.1), NTM 1b (NM_016522.2), LSAMP 1a (AK299851.1), LSAMP $1 b$ (NM_002338.3), NEGR1 (NM_173808.2) and IGLON5 (NM_001101372.2).

\section{Human Postmortem Brain Samples}

Human DLPFC tissues were obtained from the New South Wales Brain Bank Network (Sydney, Australia). The complete cohort consisted of 37 schizophrenia (including 7 schizoaffective) postmortem brain samples and 37 controls with no history of psychiatric diagnosis. Schizophrenia subjects were diagnosed according to the Diagnostic and Statistical Manual of Mental Disorders IV (DSM-4). A detailed clinical and demographic characterization of the cohort has previously been described by Weickert et al. (2010): "Detailed cohort demographic information" can be found in the Supplementary Table S5 and "Clinical characteristics of schizophrenia cohort" can be found in the Supplementary Table S6 (Weickert et al., 2010). The summary of postmortem subject demography for control and schizophrenia groups can be find in Matosin et al. (2015, Table 1). The anatomical identification and dissection of the tissue and preparation of total RNA and total protein has been described in detail in Weickert et al. (2010). Antipsychotic drug treatment premortem was standardized to lifetime

${ }^{3} \mathrm{http}: / /$ www.ebi.ac.uk/Tools/msa/clustalo/

${ }^{4}$ http://dbtss.hgc.jp/ chlorpromazine equivalent for each patient. Antidepressant drug treatment history was also specified on a qualitative scale (yes/no). Sixteen patients had a history of drug abuse in the past, either cannabis $(n=10)$, amphetamines $(n=4)$, opiates $(n=3)$, LSD $(n=5)$ or undefined hallucinogen $(n=1)$. Many of the patients had multi-drug abuse history. The use of the New South Wales Brain Bank Network cohort was approved by the Human Research Ethics Committees at the University of Wollongong (HE99/222) and the University of New South Wales (HREC07261). For the inter-hemisphere analysis, brain samples were collected from 7 deceased subjects with no evidence of neuropathology and psychiatric diseases by the Department of Pathological Anatomy and Forensic Medicine, University of Tartu, Estonia and the Estonian Forensic Science Institute, Tallinn, Estonia. The DLPFC was bilaterally dissected from the rostro-caudal middle part of the middle frontal gyrus (Brodmann area 46 and lateral part of area 9) by qualified pathologists under the full ethical approval from the Research Ethics Committee of the University of Tartu (Approval no 223/T-4 from human research ethics committee).

\section{Quantitative Real-Time Polymerase Chain Reaction (qRT-PCR) Analysis}

IgLON transcript levels were determined by two-step RT-qPCR (qPCR). First strand cDNA was synthesized by the use of Random Hexamer (Applied Biosystems) and SuperScript ${ }^{\mathrm{TM}}$ III Reverse Transcriptase (Invitrogen) according to the manufacturer's protocol. Quantitative TaqMan Assay with FAM-BHQ-probe was designed for the detection of human LSAMP 1a/1b, OPCML 1a/1b, NTM 1a/1b, NEGR1, and IGLON5 transcripts (see Table 1 for the primer and probe sequences). The general primer design schema was similar for the primer design for mouse IgLON transcripts described previously in Philips et al. (2015). In case of OPCML, NTM, and LSAMP universal reverse primer was combined with an alternative forward oligo specific for either $1 \mathrm{a}$ or $1 \mathrm{~b}$ transcript. The primer sequences have been mapped on the $5^{\prime}$-region of the IgLON transcripts in Figure 1. The sequences for IgLON transcripts and housekeeping gene HPRT1 have been listed in $5^{\prime}-3^{\prime}$ orientation in Table 1 . TaqMan Universal PCR Master Mix was used according to the manufacturer's protocol as reaction buffer. Two micrograms of RNA was used in $20 \mu \mathrm{l}$ end reaction for cDNA synthesis from brain tissues. Each reaction mix was divided out in $10 \mu \mathrm{l}$ quadruplicates. ABI Prism 7900HT Sequence Detection System with ABI Prism 7900 SDS 2.4.2 software (Applied Biosystems) was used for qPCR detection. qPCR data has been presented on linear scale, in form of $2^{-\Delta \Delta C T}$ (Livak and Schmittgen, 2001) where $\Delta \mathrm{CT}$ is the difference in cycle threshold (CT) between the gene of interest (FAM) and the housekeeper gene (VIC). The use of internal control gene, human HPRT1, has been optimized and used in our previous studies (Philips et al., 2010).

\section{Western Blot}

The protein extraction for New South Wales Brain Bank Network has been described in Matosin et al. (2015). For inter-hemisphere 
TABLE 1 | 1a/1b isoform specific primers for human IgLON gene transcripts optimized for the current study.

\begin{tabular}{|c|c|c|c|c|}
\hline Gene & & Forward primer & Probe sequence & Reverse primer \\
\hline \multirow[t]{2}{*}{ LSAMP } & $1 \mathrm{a}$ & 5'-TCCTGTCCCTCTTCTCATTGC-3' & 5'-AACCGAGGCACGGACAAC-3' & 5'-TTCTTGTCTTCTACAACGCACCTG-3' \\
\hline & $1 b$ & 5'-GATTGCTCTGCCTTCTTCCCAC-3' & & \\
\hline \multirow[t]{2}{*}{ NTM } & $1 \mathrm{a}$ & 5'-TGGCTGCTCTGTGTCTCTTCC-3' & 5'-CGGAGATGCCACCTT-3' & 5'-GGTGACCCGGTTGTCAATAGTG-3' \\
\hline & $1 b$ & 5'-TCTCAGGCTGCTGTTCCTTGTA-3' & & \\
\hline \multirow[t]{2}{*}{ OPCML } & $1 \mathrm{a}$ & 5'-TCGGCGACAACTGCCCTGCT-3' & & 5'-GGTTACCCGGTCATCTATGGTA-3' \\
\hline & $1 b$ & 5'-TCTCAGGCTGCTGTTCCTTGTA-3' & & \\
\hline NEGR1 & & 5'-TGCTCGAACCAGTGGCTGGC-3’' & 5'-TGGGCGGCCGTGGACAA-3' & 5'-CCCTTGGAAGCTCCATCTTCCA-3' \\
\hline $\operatorname{lgLON} 5$ & & 5'-TGGCCGTCATCAGCCGAG-3' & 5'-ACGCCACCCTCAGCTGCT-3' & 5'-TGGAGCGGTTCAGCCAGGC-3' \\
\hline HPRT1 & & 5'-GACTITGCTITCCTTGGTCAGG-3' & $5^{\prime}-\Pi \Pi \mathrm{TCACCAGCAAGCTTGCGACCTTGA-3^{ \prime }}$ & 5' -AGTCTGGCTTATATCCAACACTTCG-3' \\
\hline
\end{tabular}

5' FAM-3' BHQ probe was used for the detection of IgLON transcripts and 5' VIC-3' TAMRA probe was used for the housekeeping gene HPRT1.

\section{LSAMP 1 aCDS LSAMP 1 bCDS NTM1 aCDS NTM1bCDS OPCML1aCDS OPCML1bCDS NEGR1CDS IGLON5CDS}

LSAMP 1 aCDS LSAMP1bCDS NTM1 aCDS NTM1bCDS OPCML1aCDS OPCML1bCDS NEGR1CDS IGLON5CDS

LSAMP 1 aCDS LSAMP 1 bCDS NTM1 aCDS NTM1bCDS OPCML1aCDS OPCML1bCDS NEGR1CDS IGLON5CDS

LSAMP 1 aCDS
LSAMP1bCDS
NTM1 1 CDS
NTM1bCDS
OPCML1aCDS
OPCML1bCDS
NEGR1CDS
IGLON5CDS

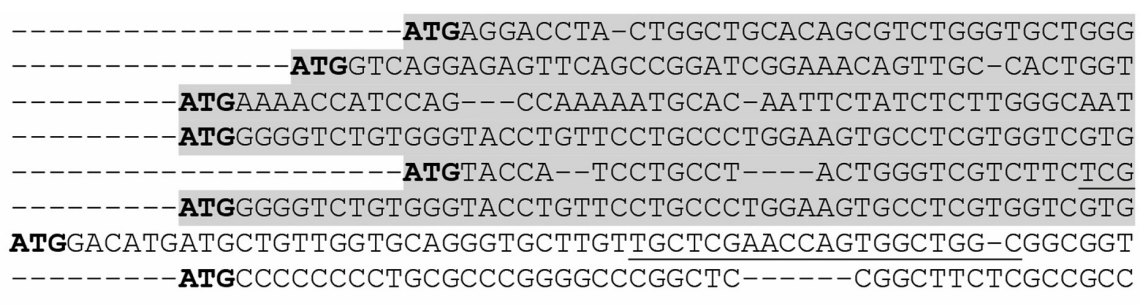

\section{forward primer binding sites}

CTTTTTCCTGTC-CCTCTTC--TCATTGCA---AGGACTGCCTGTT---CGCAGCGTGGA CCTACTGAGATT-GCTCTGCCTTCTTCCCAC--AGGACTGCCTGTT---CGCAGCGTGGA CTTCACGGGGCTGGCTGCTCTGTGTCTCTTCCAAGGAGTGCCCGTG---CGCAG EGGAGA TCTCTCAGGCT--GCTGTTCCTTGTACCCAC--AGGAGTGCCCGTG---CGCAGCGGAGA GCGACAA--CT--GCCCTGCTCTTCATCC-C--AGGAGTGCCCGTG---CGCAGCGGAGA TCTCTCAGGCT--GCTGTTCCTTGTACCCAC--AGGAGTGCCCGTG---CGCAGCGGAGA GCTCCTCAGCCT-GTGCTGCCTGCTACCCTC--CTGCCTCCCGGCTGGACAGAGTGTGGA GCCGCCCTGGCCGGCTTGGCCGT-CATCAGCCGAGGGCTGCTCTCC---CAGAGCCTGGA

TTTT AACCGAGG------CACGGACAAC ATCACCGTGAGGCAGGGGGACACAGCCATCCT TTTT AACCGAGG-----CACGGACAACATCACCGTGAGGCAGGGGGACACAGCCATCCT TGCCACCTT CCCCAAAGCTATGGACAACGTGACGGTCCGGCAGGGGGAGAGCGCCACCCT TGCCACCTT CCCCAAAGCTATGGACAACGTGACGGTCCGGCAGGGGGAGAGCGCCACCCT TGCCACCTT CCCCAAAGCTATGGACAACGTGACGGTCCGGCAGGGGGAGAGCGCCACCCT TGCCACCTT CCCCAAAGCTATGGACAACGTGACGGTCCGGCAGGGGGAGAGCGCCACCCT CTTCCCQTGGGCG---GCCGTGGACAACATGATGGTCAGAAAAGGGGACACGGCGGTGCT GTTCAACTCTCC-----TGCCGACAACTACACAGTGTGTGAAGGTGACA $\longdiv { \text { ACGCCACCCT } }$ *

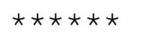

Exon 1-2

junction reverse primer binding sites

CAG IGTGCGTTGTAGAAGACAAGAACTCAAAGGTGGCCTGGTTGAACCGTTCTGGCACAG GTGCGTTGTAGAAGACAAGAACTCAAAGGTGGCCTGGTTGAACCGTTCTGGCACAG GTGCACTATTGACAACCGGGTCACCCGGGTGGCCTGGCTAAACCGCAGCACCACA ITGCACTATTGACAACCGGGTCACCCGGGTGGCCTGGCTAAACCGCAGCACCACAG IGTGTACCATAGATGACCGGGTAACCCGGGTGGCCTGGCTAAACCGCAGCACCACAG |GTGTACCATAGATGACCGGGTAACCCGGGTGGCCTGGCTAAACCGCAGCACCATAG JTGTTATTTGGAAGATGGAGCTTCAAAGGGTGCCTGGCTGAACCGGTCAAGTA-

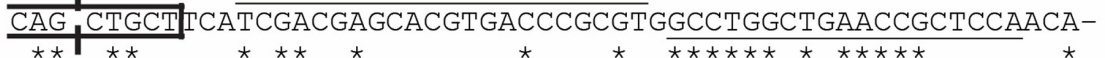

FIGURE 1 | Multiple sequence alignment of the $5^{\prime}$ coding regions (CDS) of human IgLON gene family. The unique $5^{\prime}$-regions that are distinct in $1 \mathrm{a} / 1 \mathrm{~b}$ isoforms have been highlighted with gray shading. The binding sequences for FAM-BHQplus probes have been surrounded by a box. The binding sequences for $1 \mathrm{a} / 1 \mathrm{~b}$ isoform specific primers for human IgLON gene transcripts, have been underlined. The NCBI accession numbers of the human IgLON transcripts used as an input for CLUSTALW: OPCML1a (NM_001012393.1), OPCML1b (NM_002545.3) NTM 1a (NM_001048209.1), NTM 1b (NM_016522.2), LSAMP 1a (AK299851.1), LSAMP 1b (NM_002338.3), NEGR1 (NM_173808.2) and IGLON5 (NM_001101372.2). An * (asterisk) indicates positions which have a single, fully conserved nucleotide. 
analysis, frozen tissues were sonicated in ice cold RIPA buffer (Thermo Fisher Scientific) supplemented with protease inhibitor (Life Technologies). Protein lysates were centrifuged for $10 \mathrm{~min}$ at $12,000 \mathrm{~g}$ at $4^{\circ} \mathrm{C}$. Supernatant was collected and protein concentration was determined by BCA method (Pierce BCA Protein Assay Kit, Thermo Scientific). Samples were frozen at $-80^{\circ} \mathrm{C}$ for long-term storage. NuPAGE Electrophoresis System (Life Technologies) components and equipment were used according to the manufacturer's instructions. Equal amounts of protein $(20 \mu \mathrm{g})$ were loaded into the lanes of polyacrylamide gels. The gels were electrophoresed, followed by transfer of the protein to a nitrocellulose membrane using the NuPAGE Electrophoresis System (Life Technologies). The membranes were then blocked with $3 \%$ dry milk in PBS and probed with primary antibodies overnight at $4^{\circ} \mathrm{C}$. Next day, immunoblots were incubated with fluorescent conjugated secondary antibodies for $1 \mathrm{~h}$ at room temperature, followed by visualization using a LI-COR Odyssey CLx system (LICOR Biotechnologies). Images were converted to grayscale and data was analyzed using Image Studio Lite v 3.1.4 (LI-COR Biotechnologies). MILLIPORE Re-Blot Plus Western Blot Strong Antibody Stripping Solution (1x) was used to strip and re-probe membranes.

Primary antibodies used: mouse anti-NEGR1 (1:1,000) (sc-393293, Santa Cruz), mouse anti-Ntm (1:1,000) (sc-390941, Santa Cruz), rabbit anti-GAPDH antibody (1:10,000) (247002, Synaptic Systems). Secondary antibodies used: goat anti-rabbit antibody (1:40,000) (35569, Jackson ImmunoResearch) and goat anti-mouse antibody (1:5,000) (A21057, Invitrogen). Relative intensities for NEGR1 and NTM were obtained after normalization to GAPDH. Normalized values were divided by the lowest density value, so the lowest density value will be 1 . The values were then normalized to control protein.

\section{Statistical Analysis of Human Gene Expression Data}

Statistical analyses were performed with environment for statistical computing R-3.3.2 and IBM SPSS Statistics 23. Significance level was set to $p<0.05$ and data are presented as mean \pm standard error of the mean. For analyzing gene expression data, non-parametric tests were applied, because not all of the gene expression data sets were normally distributed according to the Shapiro Wilk's normality test. Mann-Whitney $U$ test was used for comparing gene expression levels between two sample groups such as diagnostic groups (patient/control), as well as gender (male/female) and hemisphere (left/right). Additionally, Mann-Whitney $U$ test was used for comparing gene expression levels within patient groups in case of binary categorical variables that were not applicable in the control group, such as antidepressant history (yes/no), concurrent major depression (yes/no), history of suicide attempts (yes/no) and death as a result of completed suicide (yes/no). Kruskal-Wallis test was used for comparing gene expression in more than two subgroups (diagnosis with and without schizoaffective disease vs. controls, agonal state, cause of death). Post hoc Tamhane's test was carried out in order to determine the difference of groups averages more specifically. Spearman's correlations were used to determine whether sample characteristics (tissue $\mathrm{pH}$ and RIN, post mortem interval, brain weight and volume and age at death) were associated with gene expression measures. Additional measures of disease characteristics were correlated specifically with the schizophrenia group (lifetime chlorpromazine equivalent reflecting antipsychotic drug history, age of disease onset and duration of illness). Analysis of covariance (ANCOVA) for diagnostic effects on protein expression was performed, accounting for factors that were associated with gene transcript levels, as determined by the Spearman's correlations. Analyses of covariance were carried out only for NTM 1b expression data, which was distributed close to normal distribution (absolute values of skewness and kurtosis were less than 1) and the parametric approach was justified. A Chi-square test of independence was calculated, comparing the frequency of drug abuse in the groups of patients who committed suicide attempts or completed suicide and patients who did not. Binary logistic regression analysis was performed to assess the contribution of different gene expression levels to the model predicting the likelihood of being a patient. ROC analysis was performed to estimate predictive accuracy of the logistic regression model.

\section{RESULTS}

\section{In Silico Analysis of Human NEGR1 Gene and Protein}

In silico analysis of the human NEGR1 transcript with $5^{\prime} \mathrm{UTR}$ region and ESTs present in the database shows no evidence of twin promoter structure in NEGR1. Our analysis showed a single $\mathrm{CpG}$ island present in the human NEGR1 gene corresponding to the $5^{\prime} \mathrm{UTR}$ region of annotated variants NM_173808 (ENST00000357731.9). Additional EST analysis confirmed uniform $5^{\prime} \mathrm{UTR}$ region indicating single promoter structure for the uniform $5^{\prime}$-region of human NEGR1 transcript encoding for single $\mathrm{N}$-terminal signal peptide. Multiple sequence alignment of the $5^{\prime}$-regions of human IgLON gene family has been presented in Figure 1.

\section{Gene Expression Analysis in Patients in Comparison with Healthy Controls}

The qPCR data from two subjects (one from the patient group and another from the control group) was excluded from the analysis due to insufficient quality of the reactions, therefore data from 36 patients to 36 control subjects was included in the gene expression studies. There was no effect of gender or hemisphere on the expression levels of IgLON transcripts according to the Mann-Whitney $U$ test. The average gene expression values of patients were greater than averages of controls for all genes (except for IGLON5, Table 2 and Figure 2). Mann-Whitney $U$ test revealed statistically significant differences between patient and control groups only for NTM $1 \mathrm{~b}$ and NEGR1. NTM $1 \mathrm{~b}$ was significantly increased in schizophrenia subjects $(m=0.94)$ 
compared to controls $(m=0.64)(p<0.001)$ (Figure 2D), and NEGR1 was also significantly increased in schizophrenia subjects $(m=6.07)$ compared to controls $(m=4.57)(p<0.001)$ (Figure 2G).

When the patients with schizoaffective subtype $(n=7)$ of schizophrenia were separated from the patient group (Figure 2I), the Kruskal-Wallis test revealed a statistically significant difference in average expression values of NTM $1 \mathrm{~b}$ between the groups $\left(H^{2}=9.11\right.$, $\left.\mathrm{df}=2, p=0.011\right)$. Tamhane's post hoc comparisons indicated that the mean score of patients with schizophrenia $(m=0.93$, $s d=0.48)$ was statistically higher $(p<0.05)$ than the mean score of controls $(m=0.65, s d=0.39)$. Although the mean score of patients with schizoaffective diagnosis $(m=0.99, s d=0.46)$ was also higher than the mean of control subjects, the expression values of NTM $1 \mathrm{~b}$ in the schizoaffective group were significantly different from neither controls $(p=0.15)$ nor the group of patients without the schizoaffective diagnosis $(p=1)$. Similarly, the Kruskal-Wallis test revealed a statistically significant difference in NEGR1 levels between the groups when the patients with a schizoaffective subtype were separated from the patient group $\left(H^{2}=12.09\right.$, $\mathrm{df}=2, p=0.002$ ) (Figure 2J). Post hoc comparisons using Tamhane's test indicated that the mean score of patients with schizophrenia ( $m=6.09$, $s d=2.24$ ) was statistically higher $(p<0.01)$ than the mean score of controls ( $m=4.57, s d=1.47)$, but the mean score of patients with a schizoaffective diagnosis ( $m=5.97, s d=1.13$ ) did not differ significantly from the mean scores of patients without schizoaffective schizophrenia $(p=1)$; the difference between schizoaffective patients and controls showed a strong tendency toward statistical significance $(p=0.06)$.

Non-parametric correlation coefficient was used to determine whether sample characteristics were associated with gene expression measures. In the complete study group, the RIN of the sample was correlated with LSAMP 1a $(r=-0.40, p<0.01)$, LSAMP 1b $(r=-0.33, p<0.01)$, and NTM 1a $(r=-0.49$, $p<0.01)$ mRNA expression levels. Additionally, in the complete study group, $\mathrm{pH}$ of the tissue sample correlated with LSAMP 1a $(r=-0.50, p<0.01)$; LSAMP 1b $(r=-0.49, p<0.01)$, NTM 1a $(r=-0.43, p<0.01)$ and NTM 1b $(r=-0.26$, $p<0.05)$ mRNA expression levels. The ANCOVA analysis,

TABLE 2 | Averages and standard deviations of groups of patients and controls.

\begin{tabular}{|c|c|c|c|c|}
\hline & \multicolumn{2}{|c|}{ Average (standard deviation) } & \multirow[t]{2}{*}{$w$} & \multirow[t]{2}{*}{$P$-value } \\
\hline & Patients $(n=36)$ & Controls $(n=36)$ & & \\
\hline LSAMP 1a & $3.66(1.10)$ & $3.48(1.00)$ & 548 & ns \\
\hline LSAMP 1b & $3.25(1.47)$ & $3.19(1.55)$ & 598 & ns \\
\hline NTM 1a & $3.70(2.59)$ & $3.16(1.86)$ & 574 & ns \\
\hline NTM 1b & $0.94(0.47)$ & $0.64(0.39)$ & 377 & $p<0.01$ \\
\hline OPCML 1a & $3.89(1.42)$ & $3.50(0.99)$ & 522 & ns \\
\hline OPCML 1b & $0.41(0.30)$ & $0.38(0.24)$ & 567 & ns \\
\hline NEGR1 & $6.07(2.07)$ & $4.57(1.45)$ & 320 & $p<0.001$ \\
\hline IGLON5 & $0.87(0.41)$ & $0.87(0.35)$ & 658 & ns \\
\hline
\end{tabular}

W statistic from Mann-Whitney test. ns: not significant $(p>0.05)$. accounting for tissue $\mathrm{pH}$ that was found to be correlated with gene expression measures, revealed that a significant difference in NTM $1 \mathrm{~b}$ average values (patients vs. controls) was maintained after covarying for $\mathrm{pH}$ in the prefrontal cortex. More specifically, the total ANCOVA model was statistically significant $(p<0.01$, $\left.\eta_{\mathrm{p}}^{2}=0.16\right)$, and the averages of NTM $1 \mathrm{~b}$ in patients and controls differed significantly $(p<0.01)$; the value of tissue $\mathrm{pH}$ was also significant in the model $(p<0.04)$.

Logistic regression was performed in order to assess the contribution of variables to the model predicting the status of being a patient. The dichotomous variable of being or not being a patient was used as dependent variable (Table 3). According to the model, the log of the odds of being a schizophrenia patient was positively related to the expression levels of NTM $1 \mathrm{~b}$ and NEGR1 (in both cases $p<0.05$ ). In other words, the higher the NTM $1 \mathrm{~b}$ expression, the greater the odds for belonging to the patient group (3.65 times greater odds), and also, the higher the NEGR1 expression level, the greater the odds of being a patient (1.61 times). We also computed size of the area under the ROC curve. It was 0.79 showing that predictive accuracy of our logistic regression model is good ( $\mathrm{ROC}$ curve has been visualized on the Supplementary Figure S4).

\section{Analysis within Patient Group}

No significant correlations were found between gene expression values and measures of disease characteristics in the schizophrenia group, such as lifetime chlorpromazine equivalent reflecting antipsychotic drug history, age of disease onset and duration of illness. Analysis of gene expression levels within patient groups were performed in case of binary variables that were not applicable in the control group (Figure 3). Mann-Whitney $U$ test revealed a significant difference in LSAMP 1a transcript expression levels in patients with antidepressant history $(n=18, m=3.25, s d=0.53)$ and patients with no antidepressant history $(n=18, m=3.83, s d=0.96) ; w=109$, $p<0.05$; there were no other significant gene expression changes regarding antidepressant history of patients. The expression of LSAMP 1a transcript was significantly lower in patients who died as a result of completed suicide $(n=8, m=2.92, s d=0.63)$ than in patients who did not commit suicide $(n=28, m=3.87$, $s d=1.12) w=189, p<0.01$. The expression of LSAMP $1 \mathrm{~b}$ transcript was significantly lower in patients who committed a completed suicide $(n=8, m=2.21, s d=0.86)$ than in patients who did not commit suicide $(n=28, m=3.55$, $s d=1.48)$; $w=191, p<0.05$. The expression of NTM $1 \mathrm{~b}$ transcript was significantly lower in patients who committed suicide $(n=8$, $m=0.62$, $s d=0.27)$ than in patients who did not commit suicide $(n=28, m=0.97, s d=0.48) ; w=171, p<0.05$. The expression of IgLON5 transcript was significantly lower in patients who committed suicide $(n=8, m=0.56, s d=0.12)$ than in patients who did not commit suicide $(n=28, m=0.95$, $s d=0.41) ; w=181, p<0.01$; there were no other significant gene expression changes in patients who committed suicide compared with patients who did not commit suicide.

From the 37 patients in the current study cohort, 17 patients had a history of suicide attempts and 8 patients died due to completed suicide. Sixteen patients had a history of drug abuse 
A

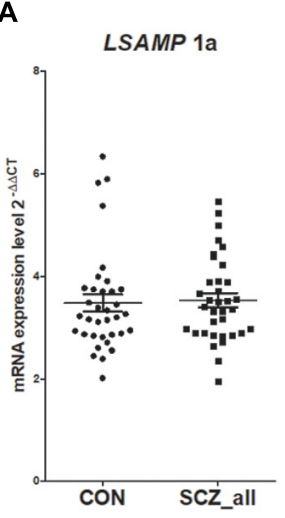

E

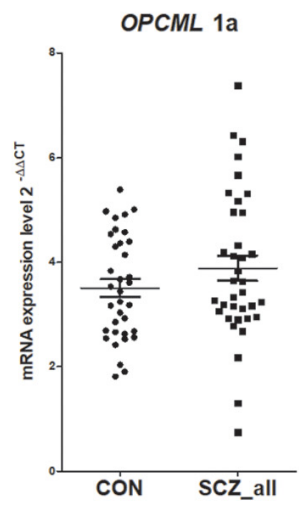

B

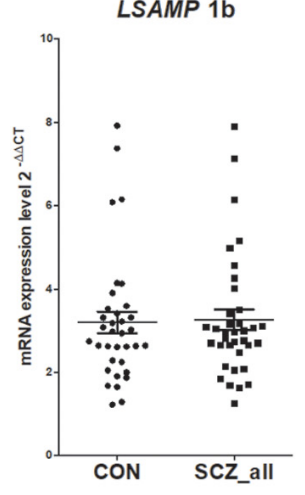

$\mathbf{F}$

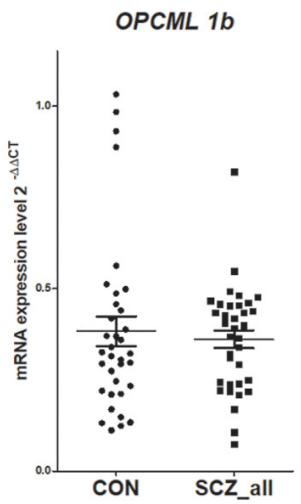

C

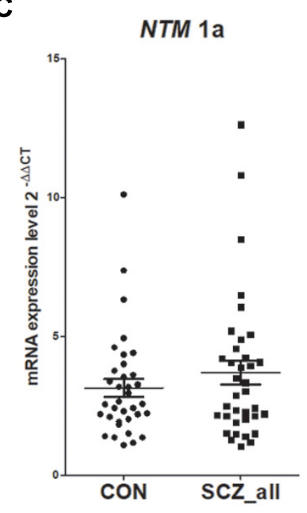

G

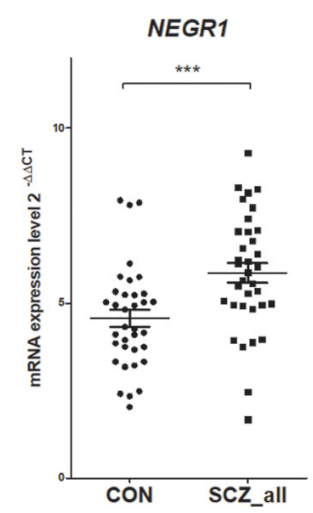

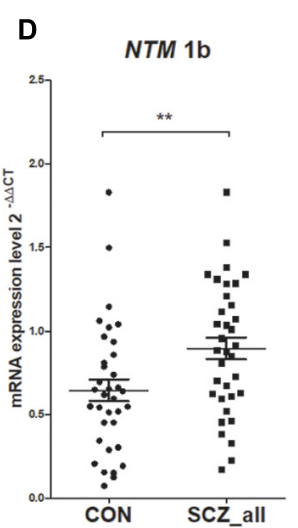

H

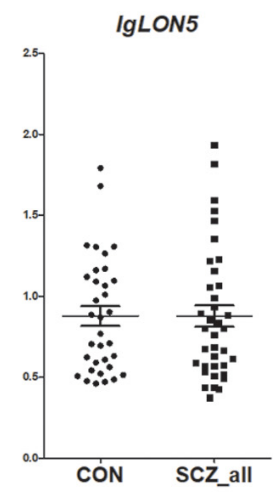

I

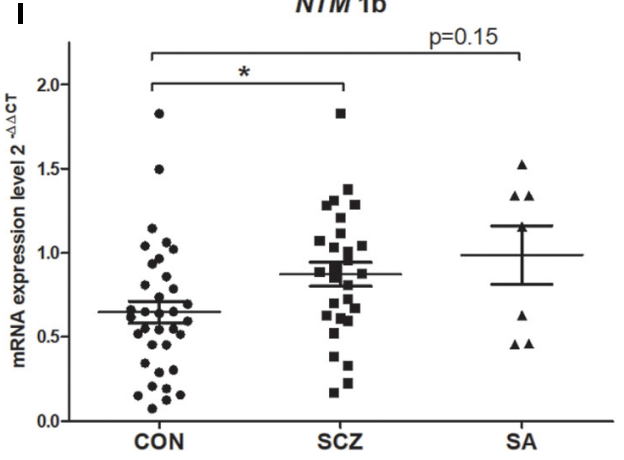

J

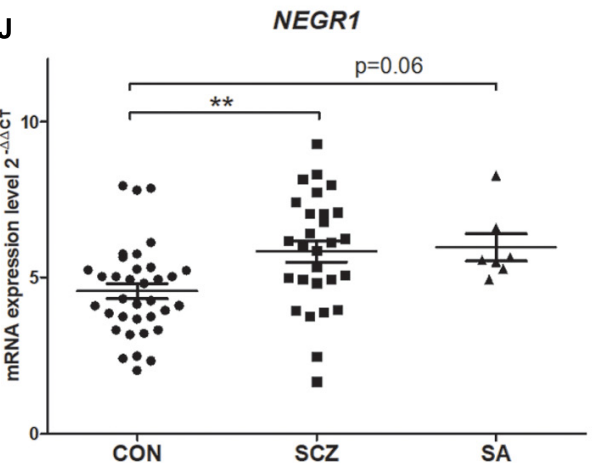

FIGURE 2 | Expression levels in the brains of controls (CON, $n=36$ ) and patients with schizophrenia (SCZ_all, $n=36$ ) for LSAMP 1a (A), LSAMP 1b (B), NTM 1a (C), NTM 1b (D), OPCML 1a (E), OPCML 1b (F), NEGR1 (G), and IgLON5 (H) transcripts. The patients with schizoaffective subtype (SA, $n=7)$ of schizophrenia were separated from the patient group (SCZ, $n=29$ ) for NTM 1b (I) and NEGR1 (J). Whiskers represent means \pm SEM. ${ }^{* * *} p<0.001,{ }^{* *} p<0.01,{ }^{*} p<0.05$ vs. control. As there was no significant difference between the gene expression values of the schizoaffective group and other schizophrenic patients $(p=1)$, we have used the data for all patients (SCZ_all) for the consequent analysis (such as logistic regression and ANCOVA).

in the past with cannabis being the most common drug used $(n=10)$. A Chi-square test of independence was calculated, comparing the frequency of drug abuse in the groups of patients who committed suicide attempts or completed suicide and who did not. A significant link was found between completed suicide and drug abuse when all drugs were included in the analysis: $c^{2}(1$, $n=37)=6.01, p<0.05$. Namely, the patients who committed suicide were more likely to have a drug abuse history ( 7 drug abusers from a total of 8 suicide cases) than patients who did not commit suicide ( 9 drug abusers from a total of 29 subjects). 
When only the use of cannabis was included in the analysis, again a significant association was found: $c^{2}(1, n=37)=9.01, p<0.01$. Namely, the patients who committed suicide were more likely to have a history of cannabis use (6 cannabis users from a total of 8 suicide cases) than patients who did not commit suicide (4 cannabis users from a total of 29 subjects). No significant association was found between suicide attempts and drug abuse when all drugs were included in the analysis: $c^{2}(1, n=37)=2.05$, $p=0.15$ (10 drug abusers from a total of 17 patients who committed suicide attempts vs. 6 drug abusers from 20 patients who had no history of suicide attempts). When only the use of cannabis was included in the analysis, a significant interaction was found: $c^{2}(1, n=37)=4.65, p<0.05$. Namely, the patients with a history of suicide attempts were more likely to have a history of cannabis use ( 8 cannabis users from a total of 17 attempters) than patients who did not commit suicide ( 2 users of cannabis from a total of 20 subjects). The amount of daily intake of ethanol showed no association with either suicidal behaviors or IgLON gene expression levels.

\section{Western Blot}

According to the Kruskal-Wallis test, there was a statistically significant difference in NEGR1 protein level between the control group and the two subgroups of schizophrenia patients $\left(H^{2}=8.01\right.$, df $=2, p=0.01$ ) (Figures 4A,B). Post hoc comparisons using Tamhane's test indicated that the mean NEGR1 protein expression score of schizophrenia patients with the paranoid subtype diagnosis $(m=1.72$, $s d=0.43$ ) was statistically higher than the mean score of controls $(m=1.24$, $s d=0.24, p<0.05)$. Additionally, the mean NEGR1 protein expression score of schizophrenia patients with another subtype diagnosis $(m=1.93, s d=0.57)$ was statistically higher than the mean score of controls $(m=1.24, s d=0.24, p<0.05)$. As the hemispheres were randomly dissected in the complete study cohort, we performed inter-hemisphere analysis in the protein level by comparatively using left and right hemisphere-derived tissue samples in 7 subjects with no evidence of neuropathology and psychiatric diseases. Inter-hemisphere analysis revealed no obvious differences in protein expression; according to Mann-Whitney $U$ test there were no significant differences in the NEGR1 and NTM proteins in the left and right hemisphere (Figures 4C-E).

\section{DISCUSSION}

Neural cell adhesion molecules, such as IgLON proteins, are critical in the formation of correct interactions between neural

TABLE 3 | Results of logistic regression model.

\begin{tabular}{lrrrr}
\hline & & \multicolumn{3}{c}{$\mathbf{9 5 \%} \mathbf{C l}$ for odds ratio } \\
\cline { 3 - 5 } & $\boldsymbol{B}(\mathrm{SE})$ & Lower & Odds ratio & Upper \\
\hline Constant & $-3.49(1.05)$ & & & \\
NTM 1b & $1.29(0.64)$ & 1.13 & 3.65 & 14.38 \\
NEGR1 & $0.49(0.18)$ & 1.16 & 1.61 & 2.38
\end{tabular}

cells that underlie normal brain function and behaviors. Here we provide evidence of altered IgLON expression profiles in the DLPFC of patients with schizophrenia. We found that elevated levels of NEGR1 transcript in the DLPFC increased the likelihood of being a patient 1.61 times; furthermore, increased levels of NTM $1 \mathrm{~b}$ transcript isoform predicted the likelihood to fall into the patient group even 3.65 times. Consequent protein analysis performed in more distinct subgroups of male subjects confirmed the increased level of NEGR1 protein both in patients with the paranoid subtype and in patients with other subtypes. Currently the largest human post mortem gene expression analysis provides supporting evidence for the upregulation of NEGR1 gene in the DLPFC area of schizophrenic patients (Fromer et al., 2016). However, this effect $(p<0.05)$ is not significant after multiple test correction. The data is still in line with our results, as whole transcriptome studies must apply stricter cut-off criteria to avoid false positive results. No evidence for differential expression of other IgLON genes, including NTM promoter specific isoforms, was provided by Fromer et al. (2016).

Interestingly, it has previously been shown that NEGR1 transcript is significantly increased in the DLPFC of human subjects with major depressive disorder compared with non-psychiatric control subjects (Chang et al., 2014); another recent publication reports a strong association of polymorfisms in the NEGR1 gene with major depression (Hyde et al., 2016). According to a genome-wide meta-analysis searching for common genetic variants influencing both schizophrenia and bipolar disorder, a polymorphism in the NTM gene (rs992564) accounts for the heritability of both disorders (Wang et al., 2010). A significant correlation between polygenic risk scores for bipolar disorder and the clinical dimension of mania in schizophrenia patients has been demonstrated recently (Ruderfer et al., 2014) expanding evidence that schizophrenia and bipolar disorder may share half of their genetic determinants (Wang et al., 2010). Elevated mean value of the NEGR1 transcript in the DLPFC of schizoaffective patients did not meet statistical significance (Figure 2J) due to a small number of schizoaffective patients in the current study $(n=7)$, however, our results together with previous evidence (Chang et al., 2014; Cox et al., 2016) suggest that increased level of NEGR1 in the frontal cortex may serve as a molecular marker for a wider spectrum of psychiatric conditions.

Our previous findings underline the importance of differentiating $1 \mathrm{a}$ and $1 \mathrm{~b}$ promoter activities when studying the IgLON family (Philips et al., 2015; Vanaveski et al., 2017). Uniform $5^{\prime}$ region of the human NEGR1 gene (Figure 1) was confirmed by in silico analysis in the current study, suggesting a single promoter similarly to the genomic structure of the mouse Negr1 gene (Vanaveski et al., 2017). The existence of putative alternative promoters predicted in public databases was not confirmed and the qPCR expression assay was designed based on human NEGR1 transcript variant NM_173808.2, which is compatible with protein variant NP_776169.2.

Surprisingly, we found no changes in the levels of LSAMP transcripts, although LSAMP protein has been found to be increased in schizophrenic patients in the DLPFC (Behan et al., 2009), anterior prefrontal cortex (Cox et al., 2016), and also 


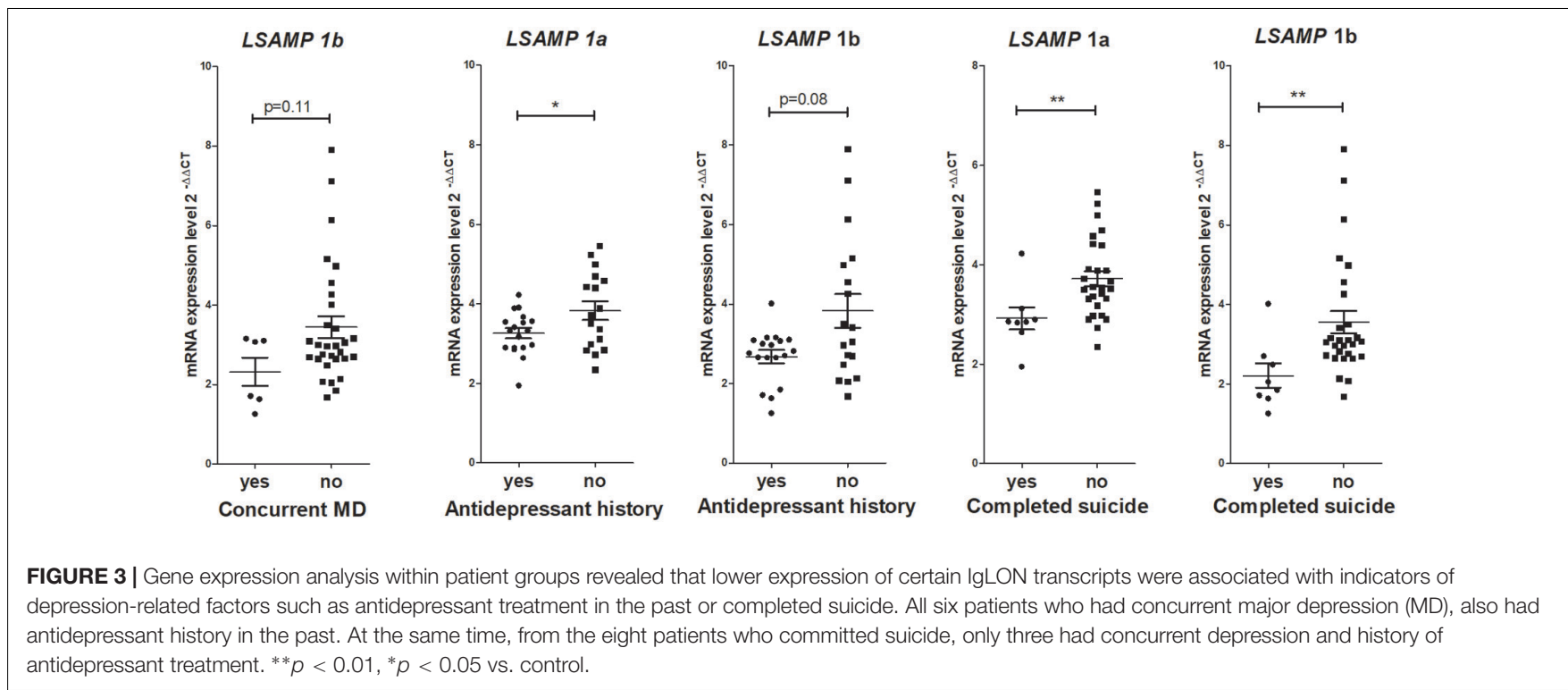

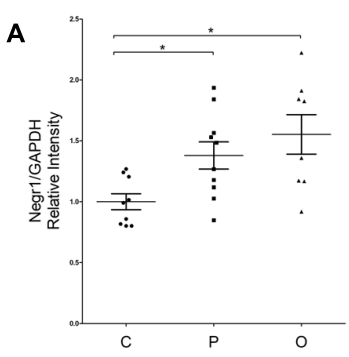

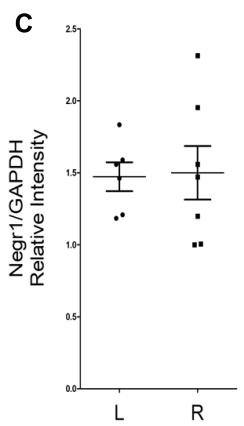

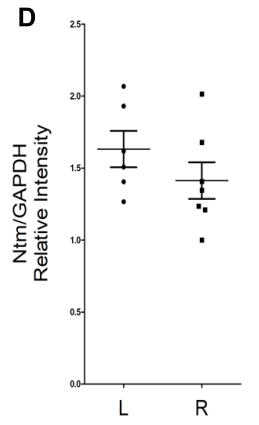

\section{B}

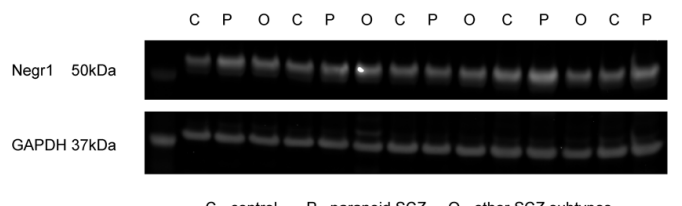

C - control P - paranoid SCZ O - other SCZ subtypes

$E$

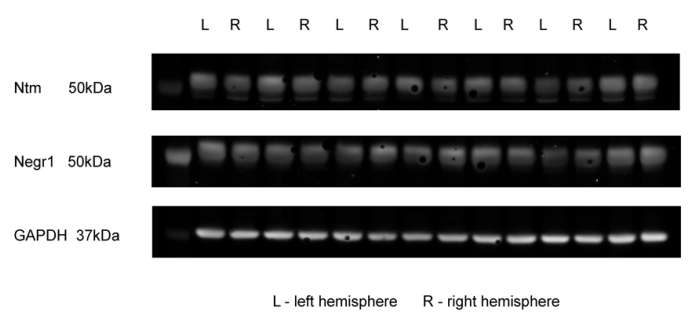

FIGURE 4 | (A,B) Analysis of NEGR1 and representative immunoblots. DLPFC samples were collected from healthy $(\mathrm{C}, n=9)$, paranoid SCZ (P, $n=10)$ and other SCZ subtype $(\mathrm{O}, n=8)$ patients. (C-E) Analysis of NEGR1 and NTM in the left $(\mathrm{L}, n=7)$ and right hemispheres $(\mathrm{R}, n=7)$, and representative immunoblots. DLPFC samples were collected from healthy patients. Values are means $\pm \mathrm{SEM}$. ${ }^{*} p<0.05$ vs. control.

the synaptosome fraction of the orbitofrontal cortex (Velásquez et al., 2017) compared to healthy controls. An explanation could be that we measured separately alternative $1 \mathrm{a}$ and $1 \mathrm{~b}$ promoter related transcripts of LSAMP that are not equivalent to the summarized level of protein in the brain. Furthermore, the presence of a conserved long $8 \mathrm{~kb}$ transcript of LSAMP has been shown (Pimenta et al., 1996), suggesting a variant with long poly-A tail which indicates that the translation levels of LSAMP may not be regulated only with the amount of mRNA. There are other studies showing that the level of mRNA might not always correspond to the level of protein, such as Matosin et al. (2015). At the same time, a significant increase of NTM 1b transcript in the DLPFC of schizophrenic patients indicates that distinguishing between alternative promoters is important and justified when studying the IgLON gene family. It is important to note that after cleavage of $\mathrm{N}$-terminal $1 \mathrm{a} / 1 \mathrm{~b}$-isoform specific signal peptides (encoded by $5^{\prime}$ - coding region, Figure 1), the mature IgLON proteins are identical regardless of the initial promoter from which the expression of particular molecule was triggered (Pimenta and Levitt, 2004). Therefore, neither LSAMP 
nor NTM antibodies are able to discriminate between 1a and $1 \mathrm{~b}$ isoforms that have highly different expressional distributions across the brain (Philips et al., 2015; Vanaveski et al., 2017) and supposedly differential roles. mRNA level analysis, such as qPCR, is the only possibility to separate promoter-specific isoforms in IgLONs.

The expression of OPCML uniform transcript has been explored in our cohort previously in a study which aimed to measure the expression levels of genes identified as schizophrenia susceptibility genes by GWASs (Umeda-Yano et al., 2014). No changes in the DLPFC of schizophrenic patients were found in the expression of OPCML uniform mRNA, similarly to our results, revealing no significant changes associated with schizophrenia in the expression of OPCML $1 \mathrm{a}$ and $1 \mathrm{~b}$ isoforms. The work of Umeda-Yano et al. (2014) provides good evidence that genomic loci which have been detected as strong genetic determinants of a particular disease in GWASs might not necessarily give rise to expressional alterations in the closest gene, and important genomic regulatory areas may span over wider chromosomal region. Human OPCML and NTM genes lie approximately $68 \mathrm{~kb}$ apart on chromosomal locus 11q25, making it probable that these genes share regulatory mechanisms. One of the example is SNP rs11222692, located in the intronic area of the NTM gene, that is eQTL influencing the expression level of the OPCML gene in the DLPFC (Fromer et al., 2016). In our current study, we found significant alterations only in the NTM $1 \mathrm{~b}$ transcript isoform. Alterations in 11q25 have already been associated with schizophrenia (Magri et al., 2010; Ye et al., 2012) and schizophrenia susceptibility has been associated with SNPs in both OPCML (O'Donovan et al., 2008; Panichareon et al., 2012) and NTM (Wang et al., 2010), making it a considerable risk locus for dysfunctional neural regulation (See also Supplementary Figure S3).

Post-psychotic depression, hopelessness, social isolation and a history of substance abuse, are the risk factors for suicide in patients with schizophrenia (Pompili et al., 2007). Interestingly, although the number of subjects was small in the current study, our study cohort provides support to the previously reported finding (Serafini et al., 2012) in that cannabis use may be a relevant risk factor associated with suicidal behaviors in psychotic patients. Further in-group analysis of patients revealed that lower expression of certain IgLON genes, mostly LSAMP transcripts, could be related with concurrent depression-related factors in schizophrenic patients (Figure 4). The expression of LSAMP $1 \mathrm{~b}$ transcript was lower in patients with concurrent major depression than in patients with no major depression, but the difference was not significant due to a small number of patients with the diagnosis of concurrent major depression $(n=6)$. Both completed suicide and antidepressant episode can be interpreted as manifestations of the depressive endophenotype, however, in the current study we can only hypothesize that lower levels of IgLONs may be implicated in concurrent depressive traits and suicidality in schizophrenic patients as our study cohort is not including depressive patients and suicide victims without schizophrenia. Also, a notable shortcoming of the study is the significant correlation of LSAMP transcripts with the $\mathrm{pH}$ of the brain tissue, which may have an effect on the results. Still, the LSAMP gene has been associated with suicide susceptibility earlier (Must et al., 2008; Sokolowski et al., 2016), and therefore IgLONs, especially LSAMP, remain important targets in suicide genetics.

The current study has several limitations which have to be pointed out. As is common for post-mortem samples, there are many variables making the samples highly heterogeneous (such as tissue $\mathrm{pH}$, brain weight and volume, RIN), which may have impact on the results. The heterogeneity of the cohort is further increased due to subjects' individual history of drug abuse and the use of other psychoactive drugs, such as antidepressants. Additionally, our patient group was characterized by significant psychopathological differences which may also result in limited statistical power. Therefore especially the results derived from the subgroup analysis must be interpreted with caution.

We have shown alterations in the expression profile of IgLON transcripts in the frontal cortex of schizophrenic patients. IgLONs have been previously associated with different aspects of normal neural circuit formation. Specifically, NEGR1 has recently been shown to regulate neuronal arborization, playing a crucial role in the proper formation of neuronal connectivity (Pischedda et al., 2014; Pischedda and Piccoli, 2016). NTM has been shown to regulate the development of the thalamocortical and pontocerebellar projections (Struyk et al., 1995; Chen et al., 2001). Earlier studies have shown that the IgLON family functions collectively in a balanced manner by the formation of highly specific complexes and interactions between IgLON family members (Reed et al., 2004). We therefore propose that alterations in the expression profile of IgLON neural adhesion molecules are associated with brain network disorganization in neuropsychiatric disorders, such as schizophrenia.

\section{CONCLUSION}

Our results provide clinical evidence beside numerous cell culture and animal experiments, confirming that certain members of the IgLON family of neural adhesion molecules, such as NEGR1, NTM, and LSAMP, are involved in finetuning of neuronal circuits underlying normal and pathological psychological/psychiatric conditions.

\section{ETHICS STATEMENT}

This study was approved by the Human Research Ethics Committees at the University of Wollongong (HE99/222) and the University of New South Wales (HREC07261) (New South Wales Brain Bank Network cohort) and Research Ethics Committee of the University of Tartu (Approval no. 223/T-4 from human research ethics committee).

\section{AUTHOR CONTRIBUTIONS}

M-AP, KK, and EV planned the study. KK and MK prepared cDNAs and performed qPCR, K-LE performed Western Blot. 
KT and JI performed statistical analysis. MJ performed in silico analysis. CW, TT, TV, JT, and MV prepared and provided brain tissues, $\mathrm{M}$-AP designed the $\mathrm{qPCR}$ assay. $\mathrm{M}-\mathrm{AP}$ and $\mathrm{KK}$ wrote the paper. KK, MK, KT, JI, MJ, MV, TT, CW, and EV participated in data interpretation and revision of the paper and all authors approved the final version.

\section{FUNDING}

This study was supported by personal investigation grant from the Estonian Research Council PUT129 (M-AP) and institutional funding from the Estonian Research Council IUT20-41 (EV) and IUT19-18 (TT). This research was also supported by the

\section{REFERENCES}

Akeel, M., McNamee, C. J., Youssef, S., and Moss, D. (2011). DIgLONs inhibit initiation of neurite outgrowth from forebrain neurons via an IgLONcontaining receptor complex. Brain Res. 1374, 27-35. doi: 10.1016/j.brainres. 2010.12.028

Baumgartner, T., Knoch, D., Hotz, P., Eisenegger, C., and Fehr, E. (2011). Dorsolateral and ventromedial prefrontal cortex orchestrate normative choice. Nat. Neurosci. 14, 1468-1474. doi: 10.1038/nn.2933

Behan, A. T., Byrne, C., Dunn, M. J., Cagney, G., and Cotter, D. R. (2009). Proteomic analysis of membrane microdomain-associated proteins in the dorsolateral prefrontal cortex in schizophrenia and bipolar disorder reveals alterations in LAMP, STXBP1 and BASP1 protein expression. Mol. Psychiatry 14, 601-613. doi: $10.1038 / \mathrm{mp} .2008 .7$

Catts, V. S., Fung, S. J., Long, L. E., Joshi, D., Vercammen, A., Allen, K. M., et al. (2013). Rethinking schizophrenia in the context of normal neurodevelopment. Front. Cell Neurosci. 7:60. doi: 10.3389/fncel.2013.00060

Chang, L. C., Jamain, S., Lin, C. W., Rujescu, D., Tseng, G. C., and Sibille, E. A. (2014). Conserved BDNF, glutamate- and GABA-enriched gene module related to human depression identified by coexpression meta-analysis and DNA variant genome-wide association studies. PLOS ONE 9:e90980. doi: 10.1371/journal. pone. 0090980

Chen, S., Gil, O., Ren, Y. Q., Zanazzi, G., Salzer, L. J., and Hillman, E. D. (2001). Ntm expression during cerebellar development suggests roles in axon fasciculation and synaptogenesis. J. Neurocytol. 30, 927-937. doi: 10.1023/A: 1020673318536

Chen, X., Long, F., Cai, B., Chen, X., and Chen, G. (2017). A novel relationship for schizophrenia, bipolar and major depressive disorder Part 3: evidence from chromosome 3 high density association screen. J. Comp. Neurol. 526, 59-79. doi: $10.1002 / \mathrm{cne} .24311$

Costain, G., and Bassett, A. S. (2012). Clinical applications of schizophrenia genetics: genetic diagnosis, risk, and counseling in the molecular era. Appl. Clin. Genet. 5, 1-18. doi: 10.2147/TACG.S21953

Cox, D. A., Gottschalk, M. G., Wesseling, H., Ernst, A., Cooper, J. D., and Bahn, S. (2016). Proteomic systems evaluation of the molecular validity of preclinical psychosis models compared to schizophrenia brain pathology. Schizophr. Res. 177, 98-107. doi: 10.1016/j.schres.2016.06.012

Fromer, M., Roussos, P., Sieberts, S. K., Johnson, J. S., Kavanagh, D. H., Perumal, T. M., et al. (2016). Gene expression elucidates functional impact of polygenic risk for schizophrenia. Nat. Neurosci. 19, 1442-1453. doi: 10.1038/nn. 4399

Giusti-Rodríguez, P., and Sullivan, P. F. (2013). The genomics of schizophrenia: update and implications. J. Clin. Invest. 123, 4557-4563. doi: 10.1172/JCI 66031

Gottesman, I. I., and Gould, T. D. (2003). The endophenotype concept in psychiatry: etymology and strategic intentions. Am. J. Psychiatry 160, 636-645. doi: 10.1176/appi.ajp.160.4.636

Harrison, P. J., and Weinberger, D. R. (2005). Schizophrenia genes, gene expression, and neuropathology: on the matter of their convergence. Mol. Psychiatry 10, 40-68. doi: 10.1038/sj.mp.4001558
European Union through the European Regional Development Fund (Project No. 2014-2020.4.01.15-0012) and H2020-MSCARISE-2016 (EU734791). CW is funded by the NSW Ministry of Health, Office of Health and Medical Research. CW is a recipient of a National Health and Medical Research Council (Australia) Principal Research Fellowship (PRF) (\#1117079).

\section{SUPPLEMENTARY MATERIAL}

The Supplementary Material for this article can be found online at: https://www.frontiersin.org/articles/10.3389/fnmol. 2018.00008/full\#supplementary-material

Hashimoto, T., Maekawa, S., and Miyata, S. (2009). IgLON cell adhesion molecules regulate synaptogenesis in hippocampal neurons. Cell Biochem. Funct. 27, 496-498. doi: 10.1002/cbf.1600

Hosak, L., Silhan, P., and Hosakova, J. (2012). Genome-wide association studies in schizophrenia, and potential etiological and functional implications of their results. Acta Medica 55, 3-11. doi: 10.14712/18059694. 2015.67

Hyde, C. L., Nagle, M. W., Tian, C., Chen, X., Paciga, S. A., Wendland, J. R., et al. (2016). Identification of 15 genetic loci associated with risk of major depression in individuals of European descent. Nat. Genet. 48, 1031-1036. doi: $10.1038 /$ ng. 3623

Koido, K., Janno, S., Traks, T., Parksepp, M., Ljubajev, Ü, Veiksaar, P., et al. (2014). Associations between polymorphisms of LSAMP gene and schizophrenia. Psychiatry Res. 215, 797-798. doi: 10.1016/j.psychres.2014.01.016

Li, P., Fan, T. T., Zhao, R. J., Han, Y., Shi, L., Sun, H. Q., et al. (2017). Altered brain network connectivity as a potential endophenotype of schizophrenia. Sci. Rep. 7:5483. doi: 10.1038/s41598-017-05774-3

Livak, K. J., and Schmittgen, T. D. (2001). Analysis of relative gene expression data using real-time quantitative PCR and the $2^{-\Delta \Delta C_{\mathrm{T}}}$ method. Methods 25, 402-408. doi: 10.1006/meth.2001.1262

Magri, C., Sacchetti, E., Traversa, M., Valsecchi, P., Gardella, R., Bonvicini, C., et al. (2010). New copy number variations in schizophrenia. PLOS ONE 5:e13422. doi: 10.1371/journal.pone.0013422

Mäki, P., Veijola, J., Jones, P. B., Murray, G. K., Koponen, H., Tienari, P., et al. (2005). Predictors of schizophrenia-a review. Br. Med. Bull. 73-75, 1-15. doi: $10.1093 / \mathrm{bmb} / \mathrm{ldh} 046$

Matosin, N., Fernandez-Enright, F., Fung, S. J., Lum, J. S., Engel, M., Andrews, J. L., et al. (2015). Alterations of mGluR5 and its endogenous regulators Norbin, Tamalin and Presol in schizophrenia: towards a model of mGluR5 dysregulation. Acta Neuropathol. 130, 119-129. doi: 10.1007/s00401-0151411-6

Must, A., Tasa, G., Lang, A., Vasar, E., Kõks, S., Maron, E., et al. (2008). Association of limbic system-associated membrane protein (LSAMP) to male completed suicide. BMC Med. Genet. 9:34. doi: 10.1186/1471-2350-9-34

Nascimento, J. M., and Martins-de-Souza, D. (2015). The proteome of schizophrenia. NPJ Schizophr. 1, 14003. doi: 10.1038/npjschz.2014.3

O'Donovan, M. C., Craddock, N., Norton, N., Williams, H., Peirce, T., Moskvina, V., et al. (2008). Identification of loci associated with schizophrenia by genome-wide association and follow-up. Nat. Genet. 40, 1053-1055. doi: $10.1038 /$ ng.201

Panichareon, B., Nakayama, K., Thurakitwannakarn, W., Iwamoto, S., and Sukhumsirichart, W. (2012). OPCML gene as a schizophrenia susceptibility locus in Thai population. J. Mol. Neurosci. 46, 373-377. doi: 10.1007/s12031011-9595-2

Philips, M. A., Kingo, K., Karelson, M., Rätsep, R., Aunin, E., Reimann, E., et al. (2010). Promoter polymorphism -119C/G in MYG1 (C12orf10) gene is related to vitiligo susceptibility and Arg4Gln affects mitochondrial entrance of Myg1. BMC Med. Genet. 11:56. doi: 10.1186/1471-2350-11-56

Philips, M. A., Lilleväli, K., Heinla, I., Luuk, H., Hundahl, C. A., Kongi, K., et al. (2015). Lsamp is implicated in the regulation of emotional and social 
behavior by use of alternative promoters in the brain. Brain Struct. Funct. 220, 1381-1393. doi: 10.1007/s00429-014-0732-x

Pimenta, A. F., Fischer, I., and Levitt, P. (1996). cDNA cloning and structural analysis of the human limbic-system-associated membrane protein (LAMP). Gene 170, 189-195.

Pimenta, A. F., and Levitt, P. (2004). Characterization of the genomic structure of the mouse limbic system-associated membrane protein (Lsamp) gene. Genomics 83, 790-801. doi: 10.1016/j.ygeno.2003.11.013

Pischedda, F., and Piccoli, G. (2016). The IgLON family member negr 1 promotes neuronal arborization acting as soluble factor via FGFR2. Front. Mol. Neurosci. 8:89. doi: 10.3389/fnmol.2015.00089

Pischedda, F., Szczurkowska, J., Cirnaru, M. D., Giesert, F., Vezzoli, E., Ueffing, M., et al. (2014). A cell surface biotinylation assay to reveal membrane-associated neuronal cues: negr1 regulates dendritic arborization. Mol. Cell. Proteomics 13, 733-748. doi: 10.1074/mcp.M113.031716

Pompili, M., Amador, X. F., Girardi, P., Harkavy-Friedman, J., Harrow, M., Kaplan, K., et al. (2007). Suicide risk in schizophrenia: learning from the past to change the future. Ann. Gen. Psychiatry 6:10. doi: 10.1186/1744-859 X-6-10

Purcell, S. M., Wray, N. R., Stone, J. L., Visscher, P. M., O’Donovan, M. C., Sullivan, P. F., et al. (2009). Common polygenic variation contributes to risk of schizophrenia and bipolar disorder. Nature 460, 748-752. doi: 10.1038/ nature 08185

Reed, J., McNamee, C., Rackstraw, S., Jenkins, J., and Moss, D. (2004). Diglons are heterodimeric proteins composed of IgLON subunits, and diglon-CO inhibits neurite outgrowth from cerebellar granule cells. J. Cell Sci. 117, 3961-3973. doi: $10.1242 /$ jcs. 01261

Ripke, S., Neale, B. M., Corvin, A., Walters, J. T., Farh, K. H., Holmans, P. A., et al. (2014). Biological insights from 108 schizophrenia-associated genetic loci. Nature 511, 421-427. doi: 10.1038/nature 13595

Ripke, S., O’Dushlaine, C., Chambert, K., Moran, J. L., Kahler, A. K., Akterin, S., et al. (2013). Genome-wide association analysis identifies 13 new risk loci for schizophrenia. Nat. Genet. 45, 1150-1159. doi: 10.1038/ng. 2742

Ruderfer, D. M., Fanous, A. H., Ripke, S., McQuillin, A., Amdur, R. L., Schizophrenia Working Group of Psychiatric Genomics Consortium et al. (2014). Polygenic dissection of diagnosis and clinical dimensions of bipolar disorder and schizophrenia. Mol. Psychiatry 19, 1017-1024. doi: 10.1038/mp. 2013.138

Salzer, J. L., Rosen, C. L., and Struyk, A. F. (1996). "GPI-anchored proteins in neural cell adhesion," in Advences in Molecular and Cellular Biology, Cell adhesion, ed. D. R. Colman (Greenwich, CT: JAI Press), 193-222.

Serafini, G., Pompili, M., Innamorati, M., Rihmer, Z., Sher, L., and Girardi, P. (2012). Can cannabis increase the suicide risk in psychosis? a critical review. Curr. Pharm. Des. 18, 5165-5187. doi: 10.2174/1381612128028 84663

Singh, T., Walters, J. T. R., Johnstone, M., Curtis, D., Suvisaari, J., Torniainen, M., et al. (2017). The contribution of rare variants to risk of schizophrenia in individuals with and without intellectual disability. Nat. Genet. 49, 1167-1173. doi: $10.1038 /$ ng.3903
Sokolowski, M., Wasserman, J., and Wasserman, D. (2016). Polygenic associations of neurodevelopmental genes in suicide attempt. Mol. Psychiatry 21, 1381-1390. doi: $10.1038 / \mathrm{mp} .2015 .187$

Stefansson, H., Ophoff, R. A., Steinberg, S., Andreassen, O. A., Cichon, S., Rujescu, D., et al. (2009). Common variants conferring risk of schizophrenia. Nature 460, 744-747. doi: 10.1038/nature08186

Struyk, A. F., Canoll, P. D., Wolfgang, M. J., Rosen, C. L., D’Eustachio, P., and Salzer, J. L. (1995). Cloning of Ntm defines a new subfamily of differentially expressed neural cell adhesion molecules. J. Neurosci. 15, 2141-2156.

Umeda-Yano, S., Hashimoto, R., Yamamori, H., Weickert, C. S., Yasuda, Y., Ohi, K., et al. (2014). Expression analysis of the genes identified in GWAS of the postmortem brain tissues from patients with schizophrenia. Neurosci. Lett. 568, 12-16. doi: 10.1016/j.neulet.2014.03.031

van Os, J., and Kapur, S. (2009). Schizophrenia. Lancet 374, 635-645. doi: 10.1016/ S0140-6736(09)60995-8

Vanaveski, T., Singh, K., Narvik, J., Eskla, K.-L., Visnapuu, T., Heinla, I., et al. (2017). Promoter-specific expression and genomic structure of IgLON family genes in mouse. Front. Neurosci. 11:38. doi: 10.3389/fnins.2017.00038

Velásquez, E., Nogueira, F. C. S., Velásquez, I., Schmitt, A., Falkai, P., Domont, G. B., et al. (2017). Synaptosomal proteome of the orbitofrontal cortex from schizophrenia patients using quantitative label-free and iTRAQ-based shotgun proteomics. J. Proteome Res. 16, 4481-4494. doi: 10.1021/acs.jproteome. $7 \mathrm{~b} 00422$

Wang, K. S., Liu, X. F., and Aragam, N. (2010). A genome-wide meta-analysis identifies novel loci associated with schizophrenia and bipolar disorder. Schizophr. Res. 124, 192-199. doi: 10.1016/j.schres.2010.09.002

Weickert, C. S., Sheedy, D., Rothmond, D. A., Dedova, I., Fung, S., Garrick, T., et al. (2010). Selection of reference gene expression in a schizophrenia brain cohort. Aust. N. Z. J. Psychiatry 44, 59-70. doi: 10.3109/00048670903393662

Ye, T., Lipska, B. K., Tao, R., Hyde, T. M., Wang, L., Li, C., et al. (2012). Analysis of copy number variations in brain DNA from patients with schizophrenia and other psychiatric disorders. Biol. Psychiatry 72, 651-654. doi: 10.1016/j. biopsych.2012.06.014

Yue, W., Yu, X., and Zhang, D. (2017). Progress in genome-wide association studies of schizophrenia in Han Chinese populations. NPJ Schizophr. 3:24. doi: 10.1038/s41537-017-0029-1

Conflict of Interest Statement: The authors declare that the research was conducted in the absence of any commercial or financial relationships that could be construed as a potential conflict of interest.

The reviewer ED and the handling Editor declared their shared affiliation.

Copyright (c) 2018 Karis, Eskla, Kaare, Täht, Tuusov, Visnapuu, Innos, Jayaram, Timmusk, Weickert, Väli, Vasar and Philips. This is an open-access article distributed under the terms of the Creative Commons Attribution License (CC BY). The use, distribution or reproduction in other forums is permitted, provided the original author(s) and the copyright owner are credited and that the original publication in this journal is cited, in accordance with accepted academic practice. No use, distribution or reproduction is permitted which does not comply with these terms. 